\title{
Directional Measures of Postural Sway Applied to the Diagnostic of Postural Stability in the Population of Adult Women with Different Body Mass Index
}

\author{
Janusz W. Błaszczyk*, Joanna Cieślińska-Świder \\ Jerzy Kukuczka Academy of Physical Education, Mikolowska str, Katowice, Poland \\ *Corresponding Author: Janusz W. Błaszczyk, Jerzy Kukuczka Academy of Physical Education, \\ Mikolowska str, Katowice, Poland, Email: j.blaszczyk@awf.katowice.pl
}

\begin{abstract}
An impact of body anthropometry on postural stability was assessed in 145 adult women with different body mass indexes (BMI). Center-of-pressure (COP) trajectories were recorded during quiet stance in two 60-second trials, first with eyes open (EO) and then with eyes closed (EC). The data were analyzed using two novel measures of postural sway, the directional indices, and stability vector, that have been proven to be reliable standards for postural stability. Results showed a significant impact of BMI and visual feedback on both the anteroposterior (DIAP) and mediolateral (DIML) directional indices. Significant changes in the stability vector azimuth ( $S V a)$ due to vision exclusion, ranged between $0.99 \pm 0.04 \mathrm{rad}(\mathrm{EO})$ and $0.96 \pm 0.05$ $\mathrm{rad}(E C)$. The SV azimuth was additionally affected by BMI $(0.8 \pm 0.05$ radians $(E O)$ and $0.66 \pm 0.05 \mathrm{rad}$ $(E C)$ in the group of women with the highest BMI). The DIML exhibited a positive correlation with BMI (0.6 and 0.71 for $E O$ and EC, respectively), while the DIAP correlated negatively with BMI (-0.55 and -0.68 for $E O$ and $E C$, respectively). In the control subjects the $S V$ magnitude i.e., the mean velocity of sway, during $E O$ trials remained at the level of $14.9 \pm 2.8 \mathrm{~mm} / \mathrm{s}$ while in the group with the highest BMI, it was reduced to 8.2 $\pm 0.8 \mathrm{~mm} / \mathrm{s}$. Both directional measures were also affected by the subjects' age. The results document the progressive decline in the postural stability control in adult women caused by excess body weight.
\end{abstract}

\section{INTRODUCTION}

Neuromechanical control of human erect posture is commonly modeled as an inverted pendulum which dynamics depend on the body's anthropometry, particularly on body height and weight. From a perspective of neuromechanics, neuromuscular control in individuals with excessive body weight is primarily determined by the universal requirement for optimization of the energetic cost of movement. This forces elimination from the behavior of the most energy-consuming movements. Additional reason for reduced motor activity in individuals with excessive body weight might be the associated decline of postural stability. This problem becomes an inspiration for the present research.

Generally, the stability of human erect posture is essential for all activities of daily living [1,2]. Unfortunately, the human upright posture itself is inherently unstable and to maintain balance it must be actively controlled [3-5]. An excessive body weight results in alteration of the inertial properties of the motor system that play a crucial role in balance recovery and thus impairs postural stability. Generally, obesity potentiates instability of the erect posture and as a result, the obese individuals are more prone to accidental falls [6]. The interaction of postural stability and motor control with its dependence on body weight recently attracted the attention of researchers and clinicians.

In humans, the stability of the upright posture is a basic property of the neuromuscular control that allows maintaining a qualitatively unchanged vertical body position in space while exposed to interferences or fluctuations, including chaotic, noise-like postural sway [5]. Postural balance and stability of human erect posture are commonly modeled as a process of active control of an inverted pendulum with the pivot point located at the ankle joints $[4,5]$. The central nervous system controls the pendulum generating force in the ankle stabilizers and in the triceps surae, mainly $[4,5,7,8]$. This redundant and nonlinear in nature control causes the human body while standing quiet to perform tiny chaotic oscillations, that are assessed in static posturography by trajectories of the center of foot pressure (COP). The COP oscillation 
characteristics are utilized to quantify the balance control and eventually for the assessment of postural stability [5,9-11]. From this perspective, it seems clear that the body's anthropometry (its height and body mass with its segmental distribution) must impact the postural sway trajectories and the overall stability of posture. The relationships between postural sway and stability of the erect posture are not direct and always should be considered in the context with the postural stability limits [5]. Additionally, as in all biological systems, we have to keep in mind that the human postural system is adaptive in nature thus the control is shaped throughout the entire life by all kinds the adaptive and compensatory adjustments [12]. For instance, in young and physically active individuals, a decline of the postural stability due to increased body weight can be, at least partially, compensated by the functional adaptation of the ankle stabilizers, the muscles that are considered as the prime-movers of the inverted pendulum [8]. Deficiency in adaptation/compensatory mechanisms, however, can deepen in chronic conditions the postural stability deficit [5].

In the literature, it is commonly claimed that postural stability in obese subjects decreases as a result of increased body sway [13]. Consequently, most posturographic studies in morbidly obese subjects documented increased sway [6, 14-25].

In contrast to the aforementioned studies, in young and physically active obese women, an unchanged or even reduced body sway has been documented [2, 26]. Such exceptional results may be explained by the specificity and homogeneity of the experimental groups in the aforementioned studies. Firstly, the subjects' young age and active lifestyle probably resulted in a fast adaptation of their muscles to excessive body weight $[2,12]$. Secondly, in young women, the gynoid type of obesity is observed that results in a lowering of the center of mass (COM) position since the adipose tissue is usually found around the hips and thighs [22]. Therefore the effective length of the inverted pendulum is shortened which slightly improves its stability. All these may explain why in young healthy women with excessive body weight decrease in postural sway can be observed [26]. These results should not be, however, compared with those obtained from individuals with morbid obesity and the resultant chronic motor deficits. Here, the essential question arises whether the results of static posturography may provide us with direct inferences for postural stability? If so, we must know which observed changes in COP trajectories can be used for the assessment of postural stability in obese subjects. Finally, we must answer the question to what extent the postural sway characteristics as observed in obese individuals may be confounded by some additional factors such as subjects' age and their BMI? Usually, it is a rather demanding task to find straightforward relationships between postural sway and the stability of the standing posture [5, 10, 11]. Recently introduced directional COP measures, including the sway directional indices [11] and the postural sway vector [5] seem to be useful and reliable measures in the assessment of postural stability. They allow for the descent assessment of the sway trajectory characteristics withing a single 60 -second trial [27]. Therefore, to get a better insight into bodyweight impact on postural balance and stability, in this research, we focused on the aforementioned sway measures. We claim that the main early predictor of the instability of the erect posture is an increase of the lateral sway. Thus, based on the previous results we put forward the hypothesis, that in young physically active women at the early phase of obesity postural balance might be well preserved.

\section{MATERial AND MethodS}

The research was accepted by the Senate Ethics Committee of the Jerzy Kukuczka Academy of Physical Education. A group of 145 adult women (mean age $37 \pm 11$ years) volunteered to participate in the study. The aim of the study and experimental procedures were explained to all subjects and written informed consent was obtained. All the participants had no evidence or known history of a gait, postural, or skeletal disorder and no history of falls. Subjects were excluded from the study if they had a balance or cardiovascular disorders, diabetes, were pregnant at the time of testing, had uncorrected vision problems, a severe musculoskeletal deformity or injury of the lower extremity that would interfere with testing.

To answer the experimental questions, the subjects participating in the studies, depending on their BMI, were assigned to five groups: control (C) consisting of 33 healthy normalweight women $(\mathrm{BMI}=22 \pm 1.8 \mathrm{~kg} / \mathrm{m} 2)$ and four groups with excessive body weight that were 
Directional Measures of Postural Sway Applied to the Diagnostic of Postural Stability in the Population of Adult Women with Different Body Mass Index

formed by 11 overweight women, 42 with grade I obesity, 29 with grade II obesity, and 30 with grade III. To estimate the length of the inverted pendulum the subjects' leg lengths were measured as a distance between the anterior superior iliac spine and the support surface. Table 1 provides the baseline characteristics of the experimental groups.

Table1: Descriptive statistics (means \pm standard deviations) and the results of ANOVA for intergroup comparisons of somatic characteristics in the examined subgroups: Normal weight, Overweight, Obesity class I, Obesity class II, Obesity class III. **-significant effect of group $P \leq .01$

\begin{tabular}{|l|l|l|l|l|l|}
\hline \multicolumn{1}{|c|}{ Group } & \multicolumn{1}{c|}{ N } & \multicolumn{1}{c|}{ AGE (yrs) } & \multicolumn{1}{c|}{ WEIGHT** } & \multicolumn{1}{c|}{ HEIGHT (m) } & \multicolumn{1}{c|}{ BMI** } \\
\hline Normal & 33 & $36 \pm 10$ & $60.1 \pm 6.5$ & $1.66 \pm 0.07$ & $21.7 \pm 1.6$ \\
\hline Overweight & 11 & $34 \pm 12$ & $77.7 \pm 5.9$ & $1.64 \pm 0.05$ & $28.6 \pm 1.4$ \\
\hline Ob. Class I & 42 & $35 \pm 13$ & $87.6 \pm 6.9$ & $1.64 \pm 0.06$ & $32.6 \pm 1.5$ \\
\hline Ob. Class II & 29 & $37 \pm 12$ & $99.7 \pm 8.3$ & $1.63 \pm 0.06$ & $37.3 \pm 1.3$ \\
\hline Ob. Class III & 30 & $40 \pm 11$ & $114.5 \pm 9.5$ & $1.62 \pm 0.07$ & $42.4 \pm 1.7$ \\
\hline
\end{tabular}

Postural sway trajectories of the participants were recorded by a force plate (Kistler 9281C) via the 16 bit analog to digital interface with a sampling frequency of $40 \mathrm{~Hz}$. Subjects were asked to stand on the force platform in a comfortable stance. Their COP trajectories were acquired in two 60-second trials: first, with eyes open (EO) and next with eyes closed (EC). The trials were separated by a short rest break to avoid fatigue or boredom. The center of footpressure AP and ML trajectories were filtered off-line at $6 \mathrm{~Hz}$ with the Chebyshev II nine order low-pass filters (Matlab v. 6.0, The MathWorks, Inc, USA).

The sway directional indices (DIAP and DIML) were computed off-line as the ratios of the anteroposterior $\left(S_{A P}\right)$ or the mediolateral $\left(S_{M L}\right)$ path lengths divided by the total COP path length $\left(S_{T O T}\right)$, respectively [11]:

$$
D I_{A P}=\frac{S_{A P}}{S_{T O T}} \quad \text { and } \quad D I_{M L}=\frac{S_{M L}}{S_{T O T}}
$$

The COP trajectories were also used to compute polar coordinates of the Sway Vector [5]:

$$
\overrightarrow{S V}=\left(S V_{m}, S V_{a}\right)
$$

Where the magnitude of the sway vector $(\mathrm{SVm})$ was equal to the mean COP velocity during the

Table2: Postural sway (COP) characteristics (means, standard deviations) in the examined groups tested while standing quietly with eyes open (EO) and eyes closed (EC), and the effect of vision and BMI group. ***-

\begin{tabular}{|c|c|c|c|c|c|c|}
\hline Group & Normal & Overweight & Class I & Class II & Class III & $F_{4,141}$ \\
\hline \multicolumn{7}{|c|}{ AP path length $(\mathrm{mm}) * * *$} \\
\hline $\mathrm{EO}$ & $442.0 \pm 72.5$ & $351.3 \pm 118.6$ & $329.9 \pm 89.3$ & $306.3 \pm 64.7$ & $292.6 \pm 46.5$ & 21.63 \\
\hline $\mathrm{EC}$ & $483.5 \pm 82.9$ & $371.9 \pm 44.1$ & $394.0 \pm 116.0$ & $399.1 \pm 90.2$ & $390.5 \pm 71.2$ & $(\mathrm{P} \leq .001)$ \\
\hline \%Change & 10.4 & 11.1 & 21.7 & 33.1 & 34.9 & \\
\hline \multicolumn{7}{|c|}{ ML path length $(\mathbf{m m}) * * *$} \\
\hline EO & $678.43 \pm 144.0$ & $464.3 \pm 101.2$ & $425.3 \pm 90.9$ & $394.5 \pm 91.2$ & $345.0 \pm 55.2$ & 110.12 \\
\hline $\mathrm{EC}$ & $698.69 \pm 148.4$ & $462.2 \pm 48.7$ & $437.2 \pm 98.4$ & $415.5 \pm 76.0$ & $355.6 \pm 45.0$ & $(\mathrm{P} \leq .001)$ \\
\hline \%Change & 3.9 & 2.0 & 3.0 & 8.1 & 4.2 & \\
\hline
\end{tabular}
significant vision effect $P \leq 001$ according to the following formula:

$$
S V a=\arctan \frac{S_{A P}}{S_{M L}}
$$

Where: $\mathrm{S}_{\mathrm{AP}}, \mathrm{S}_{\mathrm{ML}}$ are the anteroposterior and the mediolateral COP path length, respectively.

Statistical analyses were performed using Statistica 12.0 software (StatSoft Inc., Tulsa, OK, U.S.A.). The intergroup differences for somatic characteristics were tested using a oneway analysis of variance (ANOVA). The effect of BMI group on sway characteristics was assessed by repeated measures $4 \times 2$ ANOVA (BMI group $\times$ vision) followed by the LSD posthoc test. Relationships between somatic characteristics and directional sway measures were tested by Pearson's ( $r$ ) correlation. Statistical significance was defined as $\mathrm{P} \leq .05$.

\section{RESUltS}

Preliminary statistical analysis confirmed significant differences between groups in their body mass, BMI and lower extremity length. In subsequent analyses, the effects of group and visual conditions on the postural sway directional indexes were tested. The results are summarized in Table 2 . 
Directional Measures of Postural Sway Applied to the Diagnostic of Postural Stability in the Population of Adult Women with Different Body Mass Index

\begin{tabular}{|c|c|c|c|c|c|c|}
\hline $\mathrm{EO}$ & $894.96 \pm 169.0$ & $649.5 \pm 169.8$ & $600.8 \pm 131.4$ & $555.8 \pm 117.6$ & $504.0 \pm 68.8$ & 75.84 \\
\hline $\mathrm{EC}$ & $940.93 \pm 176.9$ & $664.2 \pm 59.6$ & $659.4 \pm 152.5$ & $644.5 \pm 112.6$ & $589.3 \pm 71.7$ & $(\mathrm{P} \leq .001)$ \\
\hline \%Change & 6.0 & 5.7 & 10.7 & 18.9 & 18.0 & \\
\hline \multicolumn{7}{|l|}{ DIAP $* * *$} \\
\hline EO & $0.76 \pm .03$ & $0.72 \pm .03$ & $0.71 \pm .05$ & $0.71 \pm .03$ & $0.68 \pm .05$ & 38.26 \\
\hline $\mathrm{EC}$ & $0.74 \pm .03$ & $0.70 \pm .04$ & $0.67 \pm .06$ & $0.65 \pm .06$ & $0.61 \pm .06$ & $(\mathrm{P} \leq .001)$ \\
\hline Change $\%$ & 2.1 & 3.3 & 5.7 & 8.8 & 11.3 & \\
\hline \multicolumn{7}{|c|}{ DIML $* * *$} \\
\hline $\mathrm{EO}$ & $0.50 \pm .03$ & $0.53 \pm .03$ & $0.55 \pm .05$ & $0.55 \pm .04$ & $0.58 \pm .05$ & 46.98 \\
\hline $\mathrm{EC}$ & $0.52 \pm .03$ & $0.56 \pm .04$ & $0.59 \pm .06$ & $0.62 \pm .06$ & $0.66 \pm .06$ & $(\mathrm{P} \leq .001)$ \\
\hline \%Change & 4.1 & 4.8 & 8.7 & 11.8 & 13.9 & \\
\hline \multicolumn{7}{|c|}{ SV amplitude $=$ COP Velocity $(\mathrm{mm} / \mathrm{s}) * * *$} \\
\hline $\mathrm{EO}$ & $14.9 \pm 2.8$ & $10.8 \pm 2.8$ & $10.0 \pm 2.2$ & $9.3 \pm 2.0$ & $8.4 \pm 1.1$ & 75.84 \\
\hline $\mathrm{EC}$ & $15.7 \pm 2.9$ & $11.1 \pm 1.0$ & $11.0 \pm 2.5$ & $10.7 \pm 1.9$ & $9.8 \pm 1.2$ & $(\mathrm{P} \leq .001)$ \\
\hline \%Change & 6.0 & 5.7 & 10.7 & 18.9 & 18.0 & \\
\hline \multicolumn{7}{|c|}{ SV azimuth $* * *$} \\
\hline $\mathrm{EO}$ & $0.99 \pm .04$ & $0.93 \pm .05$ & $0.92 \pm .07$ & $0.91 \pm .06$ & $0.87 \pm .08$ & 42.78 \\
\hline $\mathrm{EC}$ & $0.96 \pm .05$ & $0.89 \pm .06$ & $0.84 \pm .10$ & $0.81 \pm .09$ & $0.74 \pm .10$ & $(\mathrm{P} \leq .001)$ \\
\hline \%Change & 2.8 & 4.1 & 7.3 & 11.2 & 14.1 & \\
\hline
\end{tabular}

The ANOVA for the AP directional index Eye closure resulted in a further decrease in the $\left(D I_{A P}\right)$ confirmed a significant effect of both factors: group $\left(\mathrm{F}_{(4,140)}=25.5 ; \mathrm{P} \leq .001\right)$ and vision $\left(\mathrm{F}_{(1,140)}=100.9 ; \mathrm{P} \leq .001\right)$. The interaction of both factors was also significant $\left(\mathrm{F}_{(4,140)}=\right.$ $8.18 ; \mathrm{P} \leq .001)$. The posthoc tests documented significantly lower $D I_{A P}$ values in all groups of excessive body weight compared with the control, normal-weight group.

$D I_{A P}$ value in the obese grade I group (a decrease of about $-6 \%$, from 0.71 to 0.67 ), obese grade II (about $-9 \%$ ) and obese class III (about $11 \%)$. In the latter case, the $D I_{A P}$ decreased from $0.68 \pm 0.05$ to $0.61 \pm 0.06$ while measured with $\mathrm{EO}$ and EC, respectively (for details see Table 2 and Figure 1).

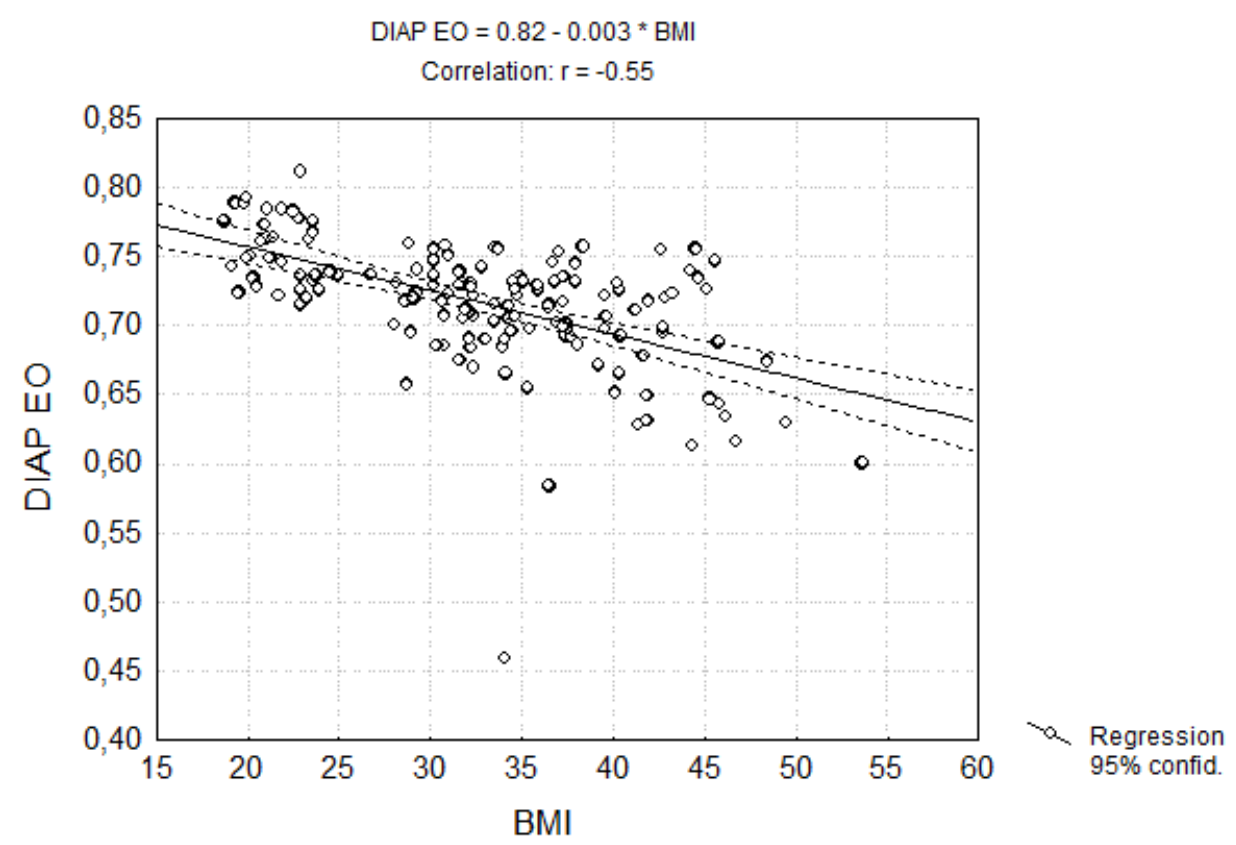

Fig1: Anteroposterior Directional Sway Indices $D I_{A P} E O$ and $D I_{A P} E C$ in obese women while standing still with eyes opened (the upper panel), and with eyes closed (the lower panel) and their dependence on BMI. Solid line represents the regression line (with $5 \%$ confidence) defined by the equations shown above each panel.

For the $D I_{M L}$, a main effect of the BMI group $\left(\mathrm{F}_{(4,140)}=30.6 ; \mathrm{P} \leq .001\right)$, vision $\left(\mathrm{F}_{(1,140)}=123.2\right.$; $\mathrm{P} \leq .001)$ and their interaction $\left(\mathrm{F}_{(4,140)}=8.3 ; \mathrm{P} \leq\right.$ .001 ) was documented (Table 2). Whereas in the control group, while standing with $\mathrm{EO}$, the $D I_{M L}$ stayed at the level $0.50 \pm .03$; in the obese grade III group, it ranged to $0.58 \pm .05$. Significantly higher $D I_{M L}$ values were observed in all experimental groups while standing with EC compared with EO. The effect of vision was, 
however, more pronounced in women with the highest BMI (Table 2). The observed increase was about $9 \%$ in obese grade I group, and $12 \%$, $14 \%$ in obese grade II, and grade III group, respectively.

Significant effects of the BMI group $\left(\mathrm{F}_{(4,140)}=\right.$ 45.2; $\mathrm{P} \leq .001)$ and vision $\left(\mathrm{F}_{(1,140)}=26.4 ; \mathrm{P} \leq\right.$ $.001)$ was documented for the Sway Vector magnitude $(S V m)$. This measure is equal to the mean COP velocity. The mean $S V m$ differed significantly between groups with excessive body weight, decreasing from $10.8 \pm 2.8 \mathrm{~mm} / \mathrm{s}$ in the 'overweight group' down to $8.4 \pm 1.1$ $\mathrm{mm} / \mathrm{s}$ in the obese grade III group (Figure 3 ). The elimination of the visual input (EC test) always resulted in an increase of the $S V m$ value within each group. The effect was the most pronounced in the obese groups (e.g., in the grade III group while measured with $\mathrm{EO}$, the $S V m$ increased from $8.4 \pm 1.1 \mathrm{~mm} / \mathrm{s}$ to $9.8 \pm 1.2$ $\mathrm{mm} / \mathrm{s}$ in the EC trial). The interaction of both factors (group x vision) did not reach, however, the 0.05 level of significance.

The BMI effect was also significant for the sway vector azimuth $(S V a)$; the main effect of the BMI group $\left(\mathrm{F}_{(4,140)}=28.2 ; \mathrm{P} \leq .001\right)$, vision $\left(\mathrm{F}_{(1,140)}=112.5 ; \mathrm{P} \leq .0001\right)$ and their interaction $\left(\mathrm{F}_{(4,140)}=8.26 ; \mathrm{P} \leq .001\right)$. A decrease of the $S V a$ was observed in response to excess body weight and the elimination of visual control $(\mathrm{p}<.001)$. For instance, in the control group, the $S V a$ stayed at the level of $0.99 \pm 0.04$ while measured with full visual control (EO trial) and slightly decreased to $0.96 \pm 0.05$ while measured with EC. In the obese group, the $S V$ azimuth negatively correlated with BMI and the effect was significantly dependent on visual control. For instance, eye closure resulted in the decline of $S V a$ that ranged: $-7 \%$ in obese grade I group, $-11 \%$ in women with obesity grade II, and even $-17 \%$ obese group grade III. For details see Table 2 and Figure 4.

Pearson's $r$ test revealed a significant correlation between directional sway indices and both body mass and BMI. The total COP path length as well as it's AP and ML components correlated negatively with body mass and BMI $(\mathrm{P} \leq .001)$. The $D I_{A P}$ negatively correlated with the BMI (Figure 1), while the BMI positively correlated with $D I_{M L}$ (Figure 2). Additionally, the $D I_{M L}$ showed significant positive correlations with subjects' age $(\mathrm{P} \leq .05)$ (for details see Table 3). Interestingly, all the directional sway measures correlated with the lower limb length but not with the body height. In particular, the leg length positively correlated with $\mathrm{DI}_{\mathrm{AP}}(0.21$ and 0.30 for $\mathrm{EO}$ and $\mathrm{EC}$, respectively) and negatively with $D I_{M L}(-0.21$ and -0.31 in EO and EC test, respectively). The values of the $D I_{A P}$ and the $D I_{M L}$ were significantly dependent on the subject's age. Whereas $D I_{A P}$ values declined with age $(\mathrm{r}=$ 0.18 and -0.23 while measured with EO and EC, respectively), the $D I_{M L}$ increased with age ( $\mathrm{r}=$ 0.19 and 0.23 for EO and EC, respectively). On the other hand, both attributes of the Sway Vector negatively correlated with BMI (for details see Figures 3 and 4).

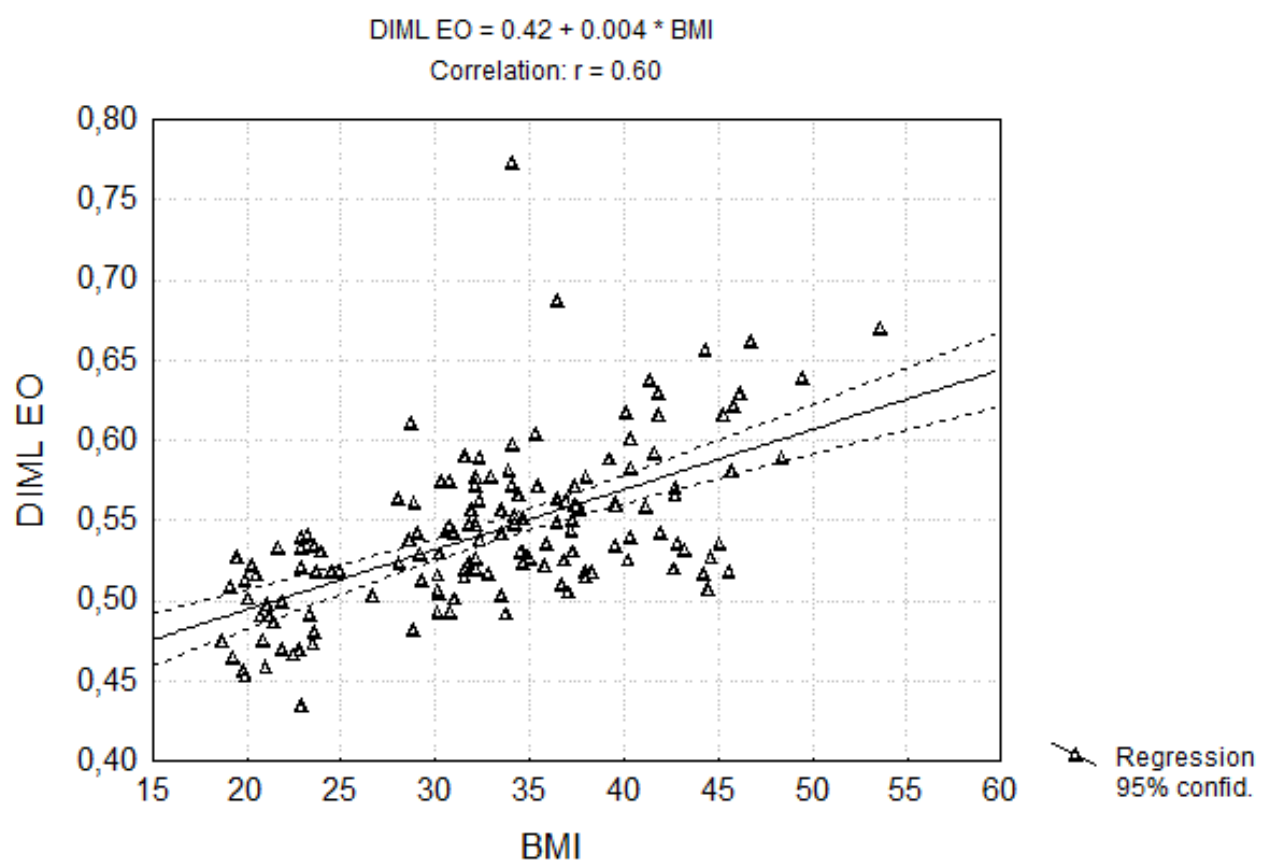


Directional Measures of Postural Sway Applied to the Diagnostic of Postural Stability in the Population of Adult Women with Different Body Mass Index

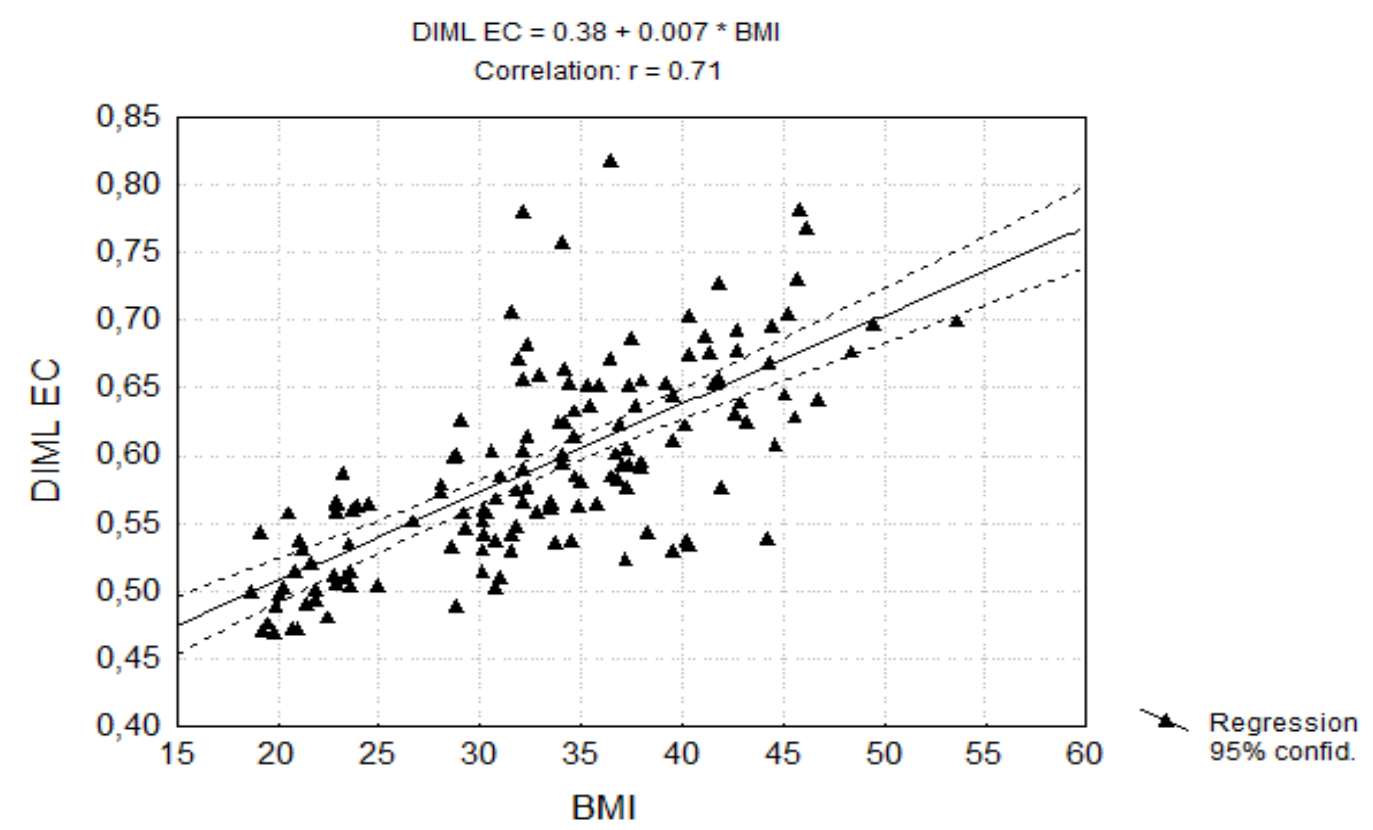

Fig2: Mediolateral Directional Sway Indices $D I_{M L} E O$ and $D I_{M L} E C$ in obese women while standing still with eyes opened (the upper panel), and then with eyes closed (the lower panel) and dependence of both measures on the BMI. Solid line represents the regression line defined by the equations shown above each panel.

Table3: Pearson's correlation coefficients $(P \leq 0.05, N=145)$ between participants' anthropometric measures and the directional sway indices (DIML and DIAP) and Sway Vector (SV) recorded with eyes open (EO) and eyes closed (EC). NS - not significant

\begin{tabular}{|c|c|c|c|c|}
\hline & AGE & HEIGHT & WEIGHT & BMI \\
\hline$D I M L(\mathrm{EO})$ & 0.19 & NS & 0.61 & 0.60 \\
\hline DIAP(EO) & -0.18 & NS & -0.56 & -0.55 \\
\hline$D I M L(\mathrm{EC})$ & 0.23 & -0.16 & 0.70 & 0.71 \\
\hline DIAP(EC) & -0.23 & 0.16 & -0.66 & -0.68 \\
\hline$S V m(\mathrm{EO})$ & NS & NS & -0.71 & -0.71 \\
\hline$S V a(\mathrm{EO})$ & -0.19 & NS & -0.51 & -0.58 \\
\hline$S V m(\mathrm{EC})$ & NS & NS & -0.63 & -0.65 \\
\hline$S V a(\mathrm{EC})$ & -0.23 & NS & -0.68 & -0.69 \\
\hline
\end{tabular}

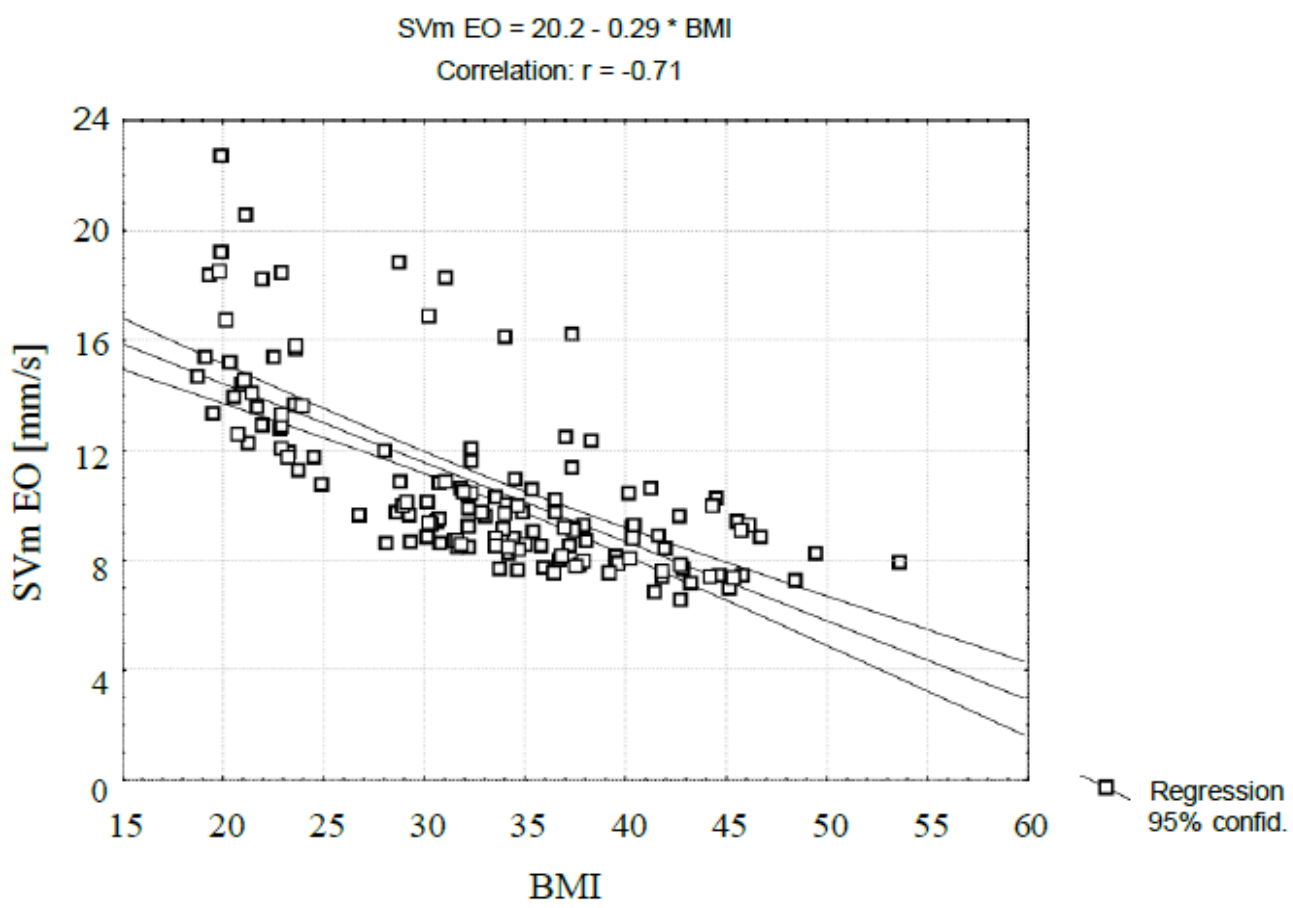


Directional Measures of Postural Sway Applied to the Diagnostic of Postural Stability in the Population of Adult Women with Different Body Mass Index

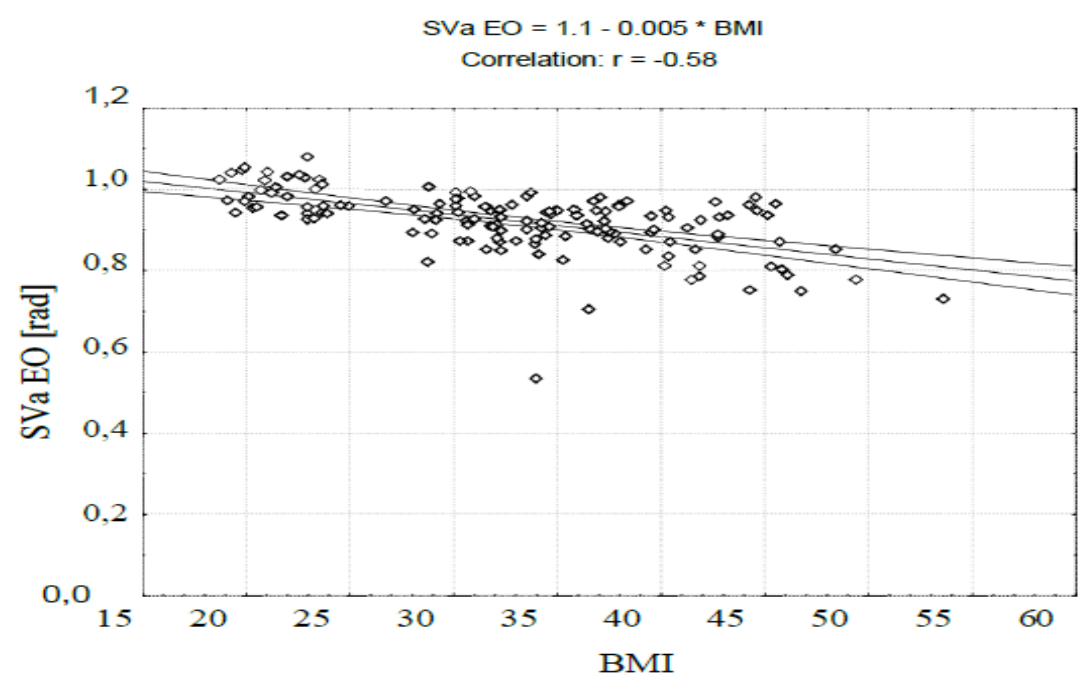

Fig3: Polar coordinates of the COP Stability Vector: its magnitude-SVm (the upper panel) and azimuth SVa (the lower panel) in obese women while standing still with their eyes opened (EO).
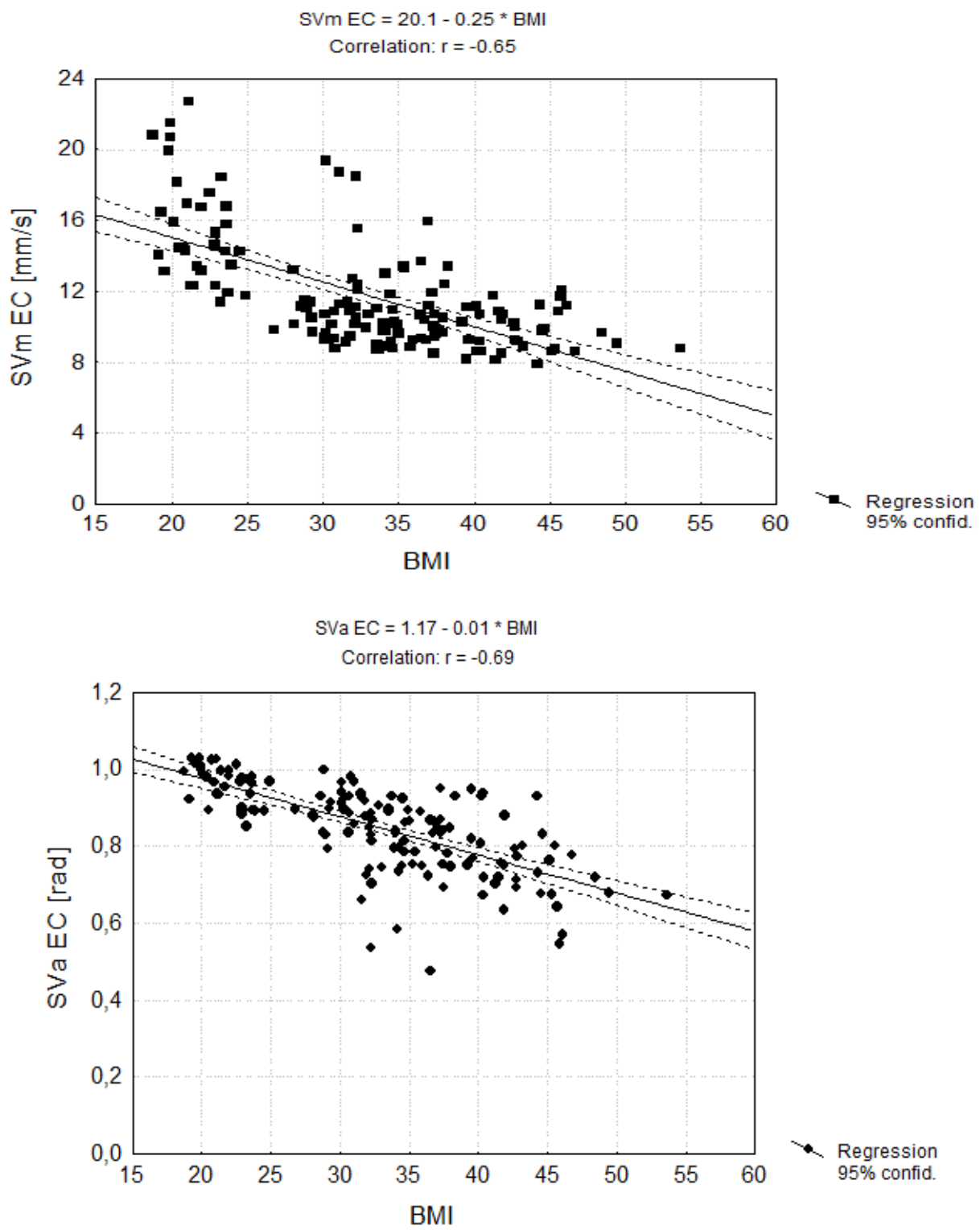

Fig4: Polar coordinates of the COP Stability Vector: its magnitude-SVm (the upper panel) and azimuth SVa (the lower panel) in obese women while standing still with their eyes closed (EC). 


\section{DISCUSSION}

Our objective was to determine how the excess of body weight impacts postural sway characteristics assessed by novel directional measures of sway trajectories. Particularly we aimed to verify whether the directional sway measures and the vector of stability would allow us to make any inferences on postural stability in adult physically active women with excessive body weight. The present results confirmed that the directional sway measures assessed in obese women differed significantly compared with normal-weight counterparts. The differences indicate only a slight decline in static balance and postural stability.

Two modes may be distinguished in the control of human posture: static equilibrium and postural stability. The equilibrium is the ability of the neuromuscular control to counterbalance gravity-derived forces and their moments during quiet stance. The postural stability, in contrast, is understood as the ability of the postural system to withstand the perturbations and efficiently recover balance. Thus it depends on both the quality of the neuromuscular control and body anthropometry, its height, and weight $[13,28,29]$. Simplifying the control of human posture to a single-link inverted pendulum model, its stability is also dependent on body mass distribution [2], which in obese individuals may lower the COM position and additionally, the size of the support surface [26,30].

Postural stability understood as the probability of successful balance recovery depends firstly on perturbation characteristics. The human body is permanently exposed to all kinds of external and internal perturbations, and the latter ones include chaotic, gravity-induced postural sway. Relatively greater postural sway should be treated as noise within the postural system which may increase uncertainty in the control. This, in turn, reduces the time for balance recovery action [5] thus increases the probability of instability and the propensity for accidental falls [26, 31].

During the upright stance, both major anatomical body planes (AP and ML) correspond to the direction of the least stability [32]. In subject standing on a force platform, the swaying movement of its center-of-mass (COM) is projected to the support surface allowing to assess its spatiotemporal characteristics based upon the center-of-pressure (COP) trajectory. The COP trajectory consists of the two components that oscillate in the middle of the support surface in two major planes, the anteroposterior (AP) and mediolateral (ML). In young healthy subjects, the ranges of these chaotic movements are relatively small to compare with stability limits [5], therefore they do not allow for direct assessment of postural stability. Only some indirect inferences may be drawn from sway characteristics by comparison of its characteristics while manipulating with sensory inputs (e.g. vision) or by displacement of the COP attractor from its central position within the base-of-support, towards a border of stability. Decrease of stability alway differently affects the postural sway and for this reason, we applied in our analysis our novel, normalized measures of postural sway. Interestingly, the increase of the ML sway is indisputably the main symptom of functional balance impairment $[5,11,33]$. In the present study, however, we observed in all obese subgroups a significant decrease in the overall postural sway, including its directional components. Despite the reduced body sway (which could be predicted as due to increased body inertia), we found rather, symptomatic changes in the directional sway characteristics that may suggest some balance deficit.

In particular, we observed a relative increase in the DIML level in women with excessive body weight. Such an increase of lateral sway index has been found to be characteristic for balance deficit in older adults [34] and Parkinsonians [33], both populations known from the progressive decline of postural stability and propensity to accidental falls. In our obese subjects the increase of $D I_{M L}$, besides a strong correlation with BMI, also positively correlated with the subject's age. Additionally, while tested with eyes closed (EC trials) a combined effect of decreased $D I_{A P}$ with simultaneous increased $D I_{M L}$ was observed. This result may support the notion that in obese individuals, similarly like in older adults, the decline in balance control is partially compensated by visual feedback [35-37].

Relevant to this discussion are changes in the natural proportion between the AP and ML COP oscillations. The stability of human upright posture is (in the inverted pendulum model) controlled in the sagittal plane by the ankle joint stabilizers [3, 4] while in the frontal plane, the control mainly relies on a hip (load/unload) mechanism [3]. Therefore, the directional sway 
measures (DIAP and DIML) or their ratio, represented here by the azimuth of stability vector (SVa), may furnish additional inferences about the status of postural control $[5,11]$. The mean COP velocity itself, represented here by the magnitude of the sway vector (SVm), does not allow the unambiguous assessment of stability. For instance, our present results showed the decline of the SVm in all obese subgroups to compare with the control. The effect was strongly dependent on BMI and would suggest an increase in postural stability due to increased body inertia [26]. Indeed, postural destabilization of an individual with a high body mass such as for instance a sumo fighter requires much higher efforts, however, if we succeed the balance recovery may be for him a great challenge. Generally, greater muscular efforts are required to recover equilibrium when the posture of participants with excessive body weight is perturbed. In erect posture, a strong correlation between the AP sway and EMG amplitude in the ankle joint stabilizers has been well documented [8, 38]. Excessive body weight (or greater BMI) can be alternatively viewed as a relative reduction in the power of the leg muscles $[12,39]$. As a consequence, it has been claimed in the literature that morbidly obese individuals exhibit a reduced capability of balance recovery due to the relative weakness of their postural muscles [2, 26, 39]. Indeed, obese women who were prone to falls had lower values of hip flexors and abductors' strength compared with age-matched non-fallers [7]. Recent studies showed also that obese individuals with balance problems had larger ankle joint stiffness and damping compared with the lean control group [12, 37].

To date studies documented that the postural balance was only slightly influenced by the anthropometric variables, both in EO and EC trials. In particular, they demonstrated that in female subjects, height explained $12 \%$ of the mediolateral COP displacements, $10 \%$ of the COP velocity, and $11 \%$ of the sway area [40]. In the present study, all experimental groups were characterized by very similar body height, all observed changes in sway characteristics can be accounted solely for the body mass and its distribution The main finding which emerged from this study was a significant increase in the
DIML index in all women with an excess of body weight. In fact, the DIML exhibit very strong correlation with bodyweight that was sensitive to visual feedback $(r=0.6(\mathrm{EO})$ and $\mathrm{r}=$ 0.7 (EC)). We should also keep in mind that DIML values might be attenuated by compensatory modification of the base of support in women with the highest BMI [41,42]. In particular, female type obesity (gynoid type) has distinct characteristics; the increased body weight is combined with a relatively larger accumulation of fat tissue in the lower limbs, especially in the hips and thighs [43]. The heavier and larger legs force an increased stance width and this, in turn, may effectively reduce the lateral sway. We observed, however, the opposite effect i.e., an increase of the DIML that correlated with body mass and the BMI. The relative increase of the lateral sway was also confirmed by the decline of the stability vector azimuth. Both observed changes (increased DIML and lower SVa) despite the decrease of the $\mathrm{SVm}$ in obese women may suggest that they develop problems with balance control $[2,18$, 44]. Additionally, women with abdominal obesity type are exposed to a greater risk of postural instability as compared to women with gynoid fat distribution [42].

In conclusion, the data presented here show, that directional measures of postural sway are superior to conventional COP parameters such as the COP path length, its velocity, or the sway area. Directional measures were differentially affected by body weight and BMI. In young and physically active women with excess body weight, the reduction of postural sway (seen in reduced COP velocity - SVm) was observed. The reduction, at least theoretically, may justify the improvement of their postural balance due to increased body inertia.

The relative increase in the mediolateral sway that was evidenced by increased DIML level and decreased SV azimuth, may imply, however, an emerging deficit in postural stability and balance control due to excess body mass and its distribution.

\section{ACKNOWLEDGMENT}

The research has been sponsored by statutory funds from the Jerzy Kukuczka Academy of Physical Education. 


\section{REFERENCES}

[1] Saibene, F., and Minetti, A.E., 2003, "Biomechanical and physiological aspects of legged locomotion in humans," Eur. J. Appl. Physiol., 88(4-5), pp. 297-316. DOI: 10.1007/s00421-002-0654-9

[2] Son, S.M., 2016, "Influence of Obesity on Postural Stability in Young Adults," Osong Public Health Res. Perspect., 7(6), pp. 378381. DOI: 10.1016/j.phrp.2016.10.001

[3] Winter, D.A., Prince, F., Frank, J.S., Powell, C., and Zabjek, K.F., 1996, "Unified theory of $\mathrm{A} / \mathrm{P}$ and $\mathrm{M} / \mathrm{L}$ balance in quiet stance," $\mathrm{J}$. Neurophysiol., 75(6), pp. 2334-2343. DOI: 10.1152/jn.1996.75.6.2334

[4] Maurer, C., and Peterka, R.J., 2005, “A new interpretation of spontaneous sway measures based on a simple model of human postural control," J. Neurophysiol., 93(1), pp. 189-200. DOI: 10.1152/jn.00221. 2004

[5] Błaszczyk, J.W., 2016, "The use of force-plate posturography in the assessment of postural instability," Gait Posture, 44, pp. 1-6. DOI: 10.1016/j.gaitpost.2015.10.014

[6] Meltzer, I., and Oddsson, L.I., 2016, “Altered characteristics of balance control in obese older adults," Obes. Res. Clin. Pract., 10(2), pp. 151158. DOI: 10.1016/j.orcp.2015.05.016

[7] Trudelle-Jackson, E.J., Jackson, A.W., and Morrow, J.R., 2006, "Muscle strength and postural stability in healthy, older women: implications for fall prevention," J. Phys. Act. Health, 3(3), pp. 292-303. DOI: 10.1123/jpah.3.3.292

[8] Borg, F., Finell, M., Herrala, M., and Hakala, I., 2007, „Analyzing gastrocnemius EMGactivity and sway data from quiet and perturbed standing," J. Electromyogr. Kinesiol., 17(5), pp. 622-634. DOI: 10.1016/j. jelekin.2006.06.004

[9] Mizrahi, J., Solzi, P., Ring, H., and Nisell, R., 2006, "Postural stability in stroke patients: vectorial expression of asymmetry, sway activity and relative sequence of reactive forces," Med. Biol. Eng. Comput., 27(2), pp. 181-190. DOI:10.1007/bf02446228

[10] Błaszczyk, J.W., 2008, "Sway ratio - a new measure for quantifying postural stability," Acta Neurobiol. Exp., 68(1), pp. 51-57. PMID:18389015

[11] Błaszczyk, J.W., Beck, M., and Sadowska, D., 2014, "Assessment of postural stability in young healthy subjects based on directional posturographic data: vision and gender effects," Acta Neurobiol. Exp. 74(4), pp. 433-442. PMID: 25576974
[12] Bollinger, L.M., 2017, "Potential contributions of skeletal muscle contractile dysfunction to altered biomechanics in obesity," Gait Posture 56, pp.100-107. DOI:10.1016/j.gaitpost.2017. 05.003

[13] Corbeil, P., Simoneau, M., Rancourt, D., Tremblay, A., and Teasdale, N., 2001, "Increased risk for falling associated with obesity: mathematical modeling of postural control," IEEE Trans. Neural. Sys. Rehabil. Eng., 9(2), pp. 126-136, DOI: 10.1109/7333. 928572

[14] McGraw, B., McClenaghan, B.A., Williams, H.G., Dickerson, J., and Ward, D.S., 2000, "Gait and postural stability in obese and nonobese prepubertal boys," Arch. Phys. Med. Rehabil. 81(4), pp. 484-489. DOI:10.1053/mr. 2000.3782

[15] Maffiuletti, N.A., Agosti, F., Proietti, M., Riva, D., Resnik, M., Lafortuna, C. L., and Sartorio, A., 2005, "Postural instability of extremely obese individuals improves after a body weight reduction program entailing specific balance training," J. Endocrinol. Invest. 28(1), pp. 2-7. DOI: $10.1007 / \mathrm{bf03345521}$

[16] Hue, O., Simoneau, M., Marcotte, J., Berrigan, F., Dore, J., Marceau, S., Tremblay, A., and Teasdale, N., 2007, "Body weight is a strong predictor of postural stability," Gait Posture 26(1), pp. 32-38. DOI: 10. 1016/j.gaitpost. 2006.07.005

[17] Greve, J., Alonso, A., Bordini, A.C., and Camanho, G.L., 2007, "Correlation between body mass index and postural balance," Clinics (Sao Paulo), 62(6), pp. 717-720. DOI: 10.1590 /s1807-59322007000600010

[18] Teasdale, N., Hue, O., Marcotte, J., Berrigan, F., Simoneau, M., Dore, J., Marceau, P., Marceau, S., and Tremblay, A., 2007, "Reducing weight increases postural stability in obese and morbid obese men," Int. J. Obes., 31(1), pp. 153-160. DOI: 10.1038/sj.ijo.08033 60

[19] Menegoni, F., Galli, M., Tacchini, E., Vismara, L., Cavigioli, M., and Capodaglio, P., 2009, "Gender-specific effect of obesity on balance," Obesity, 17(10), pp. 1951-1956. DOI: 10.1038/ oby.2009.82

[20] Handrigan, G.A., Berrigan, F., Hue, O., Simoneau, M., Corbeil, P., Tremblay, A., and Teasdale, N., 2012, "The effects of muscle strength on center of pressure-based measures of postural sway in obese and heavy athletic individuals," Gait Posture, 35(1), pp. 88-91. DOI:10.1016/j.gaitpost.2011.08.012

[21] Menegoni, F., Tacchini, E., Bigoni, M., Vismara, L., Priano, L., Galli, M., and Capodaglio, P., 2011, "Mechanisms underlying center of pressure displacements in obese 
subjects during quiet stance," J. Neuroeng. Rehabil., 8; 20, DOI: 10.1186/1743-0003-8-20

[22] Ku, P.X., Abu Osman, N.A., Yusof, A., Wan Abas, W.A., 2012, "Biomechanical evaluation of the relationship between postural control and body mass index," J. Biomech., 45(9), pp. 1638-1642, DOI: 10.1016/j.jbiomech.2012.03. 029

[23] King, A.C., Challis, J.H., Bartok, C., Costigan, F.A., and Newell, K.M., 2012, "Obesity, mechanical and strength relationships to postural control in adolescence," Gait Posture, 35(2), pp. 261-265. DOI: 10. 1016/j.gaitpost. 2011.09.017

[24] Dutil, M., Handrigan, G.A., Corbeil, P., Cantin, V., Simoneau, M., Teasdale, N., and Hue, O., 2013, "The impact of obesity on balance control in community-dwelling older women," Age, 35(3), pp. 883-890. DOI: 10.1007/ s11357-012-9386-X

[25] Meng, H., O’Connor, D.P., Lee, B.C., Layne, C.S., and Gorniak, S.L., 2016, "Effects of adiposity on postural control and cognition," Gait Posture, 43, pp.31-37. DOI:10.1016/j. gaitpost.2015.10.012

[26] Błaszczyk, J.W., Cieślińska-Świder, J., Plewa, M., Zahorska-Markiewicz, B., and Markiewicz, A., 2009, "Effects of excessive body weight on postural stability," J. Biomech., 42(9), pp.1295-1300. DOI: 10.1016/ j.jbiomech.2009.03.006

[27] Błaszczyk, J.W., Beck, M., Szczepańska, J., Sadowska, D., Bacik, B., Juras, G., and Słomka, K.J., 2016, "Directional measures of postural sway as predictors of balance instability and accidental falls," J. Hum. Kinet., 52, pp. 75-83, DOI: 10.1515/hukin2015-0195

[28] Kejonen, P., Kauranen, K., and Vanharanta, H., 2003, "The relationship between anthropometric factors and body-balancing movements in postural balance," Arch. Phys. Med. Rehabil., 84(1), pp. 17-22. DOI: 10. 1053/apmr.2003.50058

[29] Colné, P., Frelut, M.L., Pérès, G., and Thoumie, P., 2008, "Postural control in obese adolescents assessed by limits of stability and gait initiation," Gait Posture, 28(1), pp. 164169. DOI: 10.1016/j.gaitpost. 2007.11.006

[30] Opala-Berdzik, A., Błaszczyk, J.W., Bacik, B., Cieślińska-Świder, J., Świder, D., Sobota, G., and Markiewicz, A. 2015, "Static postural stability in women during and after pregnancy: A prospective longitudinal study," PLoS One, 10: e0124207. DOI: 10.1371/journal.pone.01 24207

[31] Kilby, M.C., Slobounov, S.M., and Newell, K.M., 2014, "Postural instability detection: aging and the complexity of spatial-temporal distributional patterns for virtually contacting the stability boundary in human stance," PLoS One, 9(10): e108905. DOI: 10.1371/journal. pone. 0108905

[32] Saripalle, S.K., Paiva, G.C., Cliett, T.C., Derakhshani, R.R., King, G.W., Lovelace, C.T., 2014, "Classification of body movements based on posturographic data," Hum. Mov. Sci., 33, pp. 238-250. DOI: 10.1016/j.humov.2013. 09.004

[33] Błaszczyk, J.W., and Orawiec, R. 2011., "Assessment of postural instability in patients with Parkinson's disease: sway ratio analysis," Hum. Mov. Sci., 30, pp. 396-404. DOI:10. 1016/j.humov.2010.07.017

[34] Maki, B.E., Holliday, P.J., and Topper, A.K., 1994, "A prospective study of postural balance and risk of falling in an ambulatory and independent elderly population," J. Gerontol., 49(2), pp. M72-84. DOI: 10.1093/geronj/ 49.2. $\mathrm{m} 72$

[35] Cruz-Gómez, N.S., Plascencia, G., VillanuevaPadrón, L.A., and Jáuregui-Renand, K. 2011, "Influence of obesity and gender on the postural stability during upright stance," Obes. Facts, 4(3), pp. 212-217. DOI: $10.1159 / 000$ 329408

[36] Mignardot, J-B., Olivier, I., Promayon, E., and Nougier, V., 2013, "Origins of Balance Disorders during a Daily Living Movement in Obese: Can Biomechanical Factors Explain Everything?," PLoS One, 8(4): e60491. DOI: 10.1371/journal.pone.0060491

[37] Simoneau, M., and Teasdale, N., 2015, "Balance control impairment in obese individuals is caused by larger balance motor commands variability," Gait Posture 41(1), pp. 203-208. DOI: 10.1016/j.gaitpost. 2014.10.008

[38] Sartorio, A., Lafortuna, C.L., Conte, G., Faglia, G., and Narici, M.V., 2001, "Changes in motor control and muscle performance after a shortterm body mass reduction program in obese subjects," J. Endocrinol. Invest., 24(6), pp. 393-398. DOI: 10.1007/BF03351039

[39] Wearing, S.C., Hennig, E.M., Byrne, N.M., Steele, J.R., and Hills, A.P., 2006, "The biomechanics of restricted movement in adult obesity," Obes. Rev. 7(1), pp. 13-24, DOI:10. 1111/j.1467-789X.2006. 00215.x

[40] Alonso, A.C., Luna, N.M., Mochizuki, L., Barbieri, F., Santos, S., and Greve, J.M., 2012, "The influence of anthropometric factors on postural balance: the relationship between body composition and posturographic measurements in young adults," Clinics (Sao Paulo), 67(12):1433-41. DOI: $10.6061 /$ clinics/2012 (12) 14

[41] Błaszczyk, J.W., Plewa, M., Cieślińska-Świder, J., Bacik, B., Zahorska-Markiewicz, B., 
Directional Measures of Postural Sway Applied to the Diagnostic of Postural Stability in the Population of Adult Women with Different Body Mass Index

Markiewicz, A., 2011, "Impact of excess body weight on walking at the preferred speed," Acta Neurobiol. Exp., 71(4), pp. 528-540, PMID: 22237498.

[42] Błaszczyk, J.W., Opala-Berdzik, A., Plewa, M., 2016, "Adaptive changes in spatiotemporal gait characteristics in women during pregnancy," Gait Posture, 43, pp. 160-164. DOI: 10.1016/j.gaitpost. 2015.09.016
[43] Cieślińska-Świder, J., Furmanek, M.P., and Błaszczyk, J.W., 2017, "The influence of adipose tissue location on postural control," J. Biomech., 60, pp. 162-169. DOI: 10.1016/ j.jbiomech.2017.06.027

[44] Gao, X., Wang, L., Shen, F., Ma, Y., Fan, Y., and Niu, H. 2019, "Dynamic walking stability of elderly people with various BMIs," Gait Posture 68, pp. 168-173. DOI: 10.1016/j. gaitpost.2018.11.027

Citation: Janusz W. Błaszczyk, Joanna Cieślińska-Świder. Directional Measures of Postural Sway Applied to the Diagnostic of Postural Stability in the Population of Adult Women with Different Body Mass Index. ARC Journal of Neuroscience. 2019; 4(2):8-19. doi: dx.doi.org/10.20431/2456-057X 0402003.

Copyright: () 2019 Authors. This is an open-access article distributed under the terms of the Creative Commons Attribution License, which permits unrestricted use, distribution, and reproduction in any medium, provided the original author and source are credited. 